\title{
Magnetic Susceptibility of Quasi-two-dimensional Cuprate Antiferromagnets
}

\author{
Milica S. Rutonjski*, Milan R. Pantić, Slobodan M. Radošević, \\ Milica V. Pavkov-Hrvojević \\ Department of Physics, Faculty of Sciences, University of Novi Sad, \\ Trg Dositeja Obradovića 4, Novi Sad, Serbia
}

Received: November 7, 2013

\begin{abstract}
Parallel magnetic susceptibility temperature dependence of the high- $T_{C}$ superconducting parent compound $\mathrm{La}_{2} \mathrm{CuO}_{4}$ is calculated in both antiferromagnetic (AFM) and paramagnetic phase. By making use of the quantum Heisenberg $S=\frac{1}{2}$ three-dimensional AFM model including the in-plane spin anisotropy, the calculation is performed within the framework of three different theories: Green's function theory in random-phase approximation (RPA), linear spinwave (LSW) theory and mean-field (MF) theory. The results suggest that at low temperatures quantum spin fluctuations play an important role, while at the temperatures above the critical one short-range correlations have a great impact on the behavior of the system. This leads to the discrepancy between RPA and MF results, since the later neglects the above phenomena. Further, LSW theory expectedly agrees with RPA results only at low temperatures where the magnon interactions are negligible. Comparison to the theoretical and experimental results quoted in literature confirms that RPA method presents the most appropriate method among the applied ones, suggesting that this approach is satisfactory in the case of the parallel magnetic susceptibility, while in order to reproduce the transversal one, spin-orbit coupling must be included.
\end{abstract}

Key words: Quasi-two-dimensional antiferromagnetic copper oxides, magnetic susceptibility, Green's function approach

\section{Introduction}

In the study of the magnetism of $\mathrm{CuO}_{2}$ planes in the quasi-two-dimensional (Q2D) cuprate antiferromagnets (AFM) important place belongs to the investigation of the magnetic susceptibility, which is mostly due to the fact that this property can be experimentally determined. Special interest is directed towards the high- $T_{C}$ superconducting parent compounds, as for instance $\mathrm{La}_{2} \mathrm{CuO}_{4}$ and $\mathrm{YBa}_{2} \mathrm{Cu}_{3} \mathrm{O}_{6}$, the thorough study of which has

\footnotetext{
${ }^{*}$ Corresponding author

Email address: milica.rutonjski@df.uns.ac.rs
} 
been actual since the discovery of the high- $T_{C}$ superconductivity [1] in order to elucidate the underlying physics of the phenomena. Taking into account that the experimental data for $\mathrm{YBa}_{2} \mathrm{Cu}_{3} \mathrm{O}_{6}$ are scarce, we focus on the magnetic susceptibility of $\mathrm{La}_{2} \mathrm{CuO}_{4}$, which has been both experimentally [2] and theoretically [3, 4] examined. The attention devoted to the determination of magnetic susceptibility in this compound is justified since the full knowledge of this property may shed light on the magnetism of doped $\mathrm{CuO}_{2}$ planes, which are assumed [2] to possess domain structure at temperatures above the critical one as well as in the spin glass phase. The theoretical models for this system given in literature mostly include Dzyaloshinsky-Moriya (DM) interaction, which arises from the spin-orbit coupling, leading to the weak ferromagnetism within the $\mathrm{CuO}_{2}$ planes. Our goal will be to employ the model with the in-plane spin anisotropy only, which the authors have already shown to reproduce correctly many of the experimentally determined magnetic properties in $\mathrm{La}_{2} \mathrm{CuO}_{4}[5,6,7,8]$, such as spin-wave spectrum, magnetization and Néel temperature. We shall try to reproduce the experimentally obtained results for the parallel magnetic susceptibility, defined as the response of the system to the external magnetic field acting along the easy-axis of magnetization, using three different approaches, namely Green's function (GF) method in the random-phase approximation (RPA), linear spinwave (LSW) theory and mean-field (MF) theory. This paper is organized as follows: in Sec. II we present the magnetic unit cell of $\mathrm{La}_{2} \mathrm{CuO}_{4}$ and propose the model Hamiltonian with the detailed description of the dominant exchange interactions. Sec. III, Sec. IV and Sec. V are devoted to the detailed calculation of the parallel magnetic susceptibility using GF method in RPA, LSW theory and MF approximation, respectively. In Sec. VI we analyze the results obtained within the framework of different theories and compare them to the results of both theoretical and experimental studies given in literature. Sec. VII summarizes our results and conclusions, concerning the domain of the applicability of the model used, as well as its limitations.

\section{Model Hamiltonian}

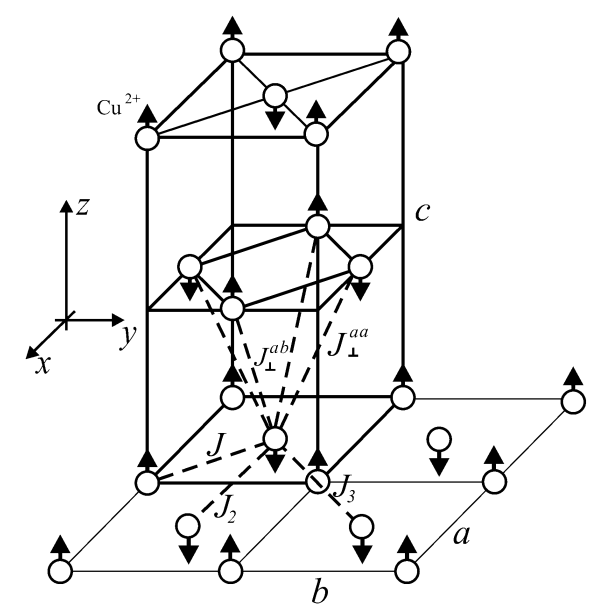

Figure 1. Magnetic unit cell of $\mathrm{La}_{2} \mathrm{CuO}_{4}$ with only $\mathrm{Cu}^{2+}$ ions shown. The arrows point in the spin direction on the given site of the crystal lattice. Dominant intra- and interplane interaction are presented by dashed lines. The parameters of the unit cell are denoted by $a, b$ and $c$. 
The magnetic structure of $\mathrm{La}_{2} \mathrm{CuO}_{4}$ is well-known $[9,10]$ : the spins from the incomplete d-shells localized on the crystal lattice sites form two magnetic sublattices and interact via exchange interactions described by integrals $J$. The neutron diffraction has shown that the spins within the $\mathrm{CuO}_{2}$ planes are AFM ordered. Magnetic structure of $\mathrm{La}_{2} \mathrm{CuO}_{4}$ in the orthorhombic phase together with the dominant exchange interactions is shown in Fig. 1.

The starting point in our considerations is the effective spin Hamiltonian, referring to the orthorhombic phase of $\mathrm{La}_{2} \mathrm{CuO}_{4}$ in the external magnetic field. In the local coordinate system, it may be written in the following form:

$$
\begin{aligned}
& \hat{H}=\sum_{\boldsymbol{n}_{a}, \boldsymbol{\delta}_{1}}\left[\frac{1}{2}\left(\hat{S}_{\boldsymbol{n}_{a}}^{+(a)} \hat{S}_{\boldsymbol{n}_{a}+\boldsymbol{\delta}_{1}}^{+(b)}+\hat{S}_{\boldsymbol{n}_{a}}^{-(a)} \hat{S}_{\boldsymbol{n}_{a}+\boldsymbol{\delta}_{1}}^{-(b)}\right)-(1+\eta) \hat{S}_{\boldsymbol{n}_{a}}^{z(a)} \hat{S}_{\boldsymbol{n}_{a}+\boldsymbol{\delta}_{1}}^{z(b)}\right]+ \\
& +\sum_{\substack{\boldsymbol{n}_{\alpha} \\
(\alpha=a, b)}} \sum_{\substack{\boldsymbol{\delta}_{i} \\
(i=2,3)}} \frac{J_{i}}{2}\left[\frac{1}{2}\left(\hat{S}_{\boldsymbol{n}_{\alpha}}^{+(\alpha)} \hat{S}_{\boldsymbol{n}_{\alpha}+\boldsymbol{\delta}_{i}}^{-(\alpha)}+\hat{S}_{\boldsymbol{n}_{\alpha}}^{-(\alpha)} \hat{S}_{\boldsymbol{n}_{\alpha}+\boldsymbol{\delta}_{i}}^{+(\alpha)}\right)+\hat{S}_{\boldsymbol{n}_{\alpha}}^{z(\alpha)} \hat{S}_{\boldsymbol{n}_{\alpha}+\boldsymbol{\delta}_{i}}^{z(\alpha)}\right]+
\end{aligned}
$$

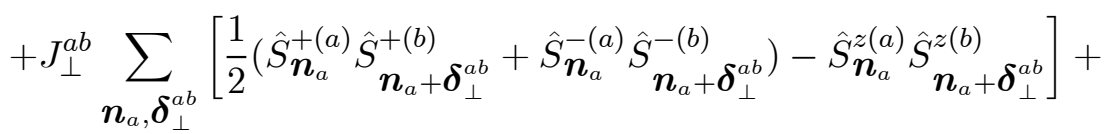

$$
\begin{aligned}
& +\sum_{\substack{\boldsymbol{n}_{\alpha} \boldsymbol{\delta}_{\perp}^{\alpha \alpha}(\alpha=a, b) \\
(\alpha)}} \frac{J_{\perp}^{\alpha \alpha}}{2}\left[\frac{1}{2}\left(\hat{S}_{\boldsymbol{n}_{\alpha}}^{+(\alpha)} \hat{S}_{\boldsymbol{n}_{\alpha}+\boldsymbol{\delta}_{\perp}^{\alpha \alpha}}^{-(\alpha)}+\hat{S}_{\boldsymbol{n}_{\alpha}}^{-(\alpha)} \hat{S}_{\boldsymbol{n}_{\alpha}+\boldsymbol{\delta}_{\perp}^{\alpha \alpha}}^{\alpha(\alpha)}+\hat{S}_{\boldsymbol{n}_{\alpha}}^{z(\alpha)} \hat{S}_{\boldsymbol{n}_{\alpha}+\boldsymbol{\delta}_{\perp}^{z \alpha}}^{\alpha \alpha}\right]+\right. \\
& -g \mu_{B} \mathcal{H}\left[\sum_{\boldsymbol{n}_{a}} \hat{S}_{\boldsymbol{n}_{a}}^{z(a)}-\sum_{\boldsymbol{n}_{b}} \hat{S}_{\boldsymbol{n}_{b}}^{z(b)}\right] .
\end{aligned}
$$

First term in (1) describes the nearest neighbor in-plane exchange interaction, where $\boldsymbol{n}_{\alpha}$ denotes the position of the given spin in the sublattice $\alpha(\alpha=a, b)$, while $\boldsymbol{\delta}_{1}$ denotes the vector connecting the given spin with its nearest neighbors in the plane. Small positive parameter $\eta$ introduces the in-plane spin anisotropy which gives rise to the long-range AFM order within the plane and defines the easy-axis of magnetization along the $+z$ axis. Second term describes the next- and next-next-nearest neighbor in-plane exchange interaction, where we assume that $J_{2}=J_{3}$ [11]. In spite of the fact that the interplane exchange interactions are much smaller in magnitude that the intraplane ones, leading to the Q2D behavior of the cuprate antiferromagnets, the detailed description of the observed compound requires including the interplanar coupling as well. Therefore, the next two terms in (1) describe the exchange interaction among the given spin and its nearest neighbors in the neighboring planes. Therein, we make the assumption that $J_{\perp}^{a a}=$ $J_{\perp}^{b b}$. The interplane interactions of longer range are rightfully neglected. All exchange interactions are assumed to be AFM in sign. Though, due to the frustration, some of the couplings are ferromagnetic, as can be seen in Fig. 1. The last term in Hamiltonian describes the interaction with the external magnetic field $\mathcal{H}$ directed along $+z$ axis.

In the above Hamiltonian it is possible to introduce the antisymmetric DM interaction, resulting from the spin-orbit coupling which causes the $\mathrm{CuO}_{2}$ planes to exhibit the weak ferromagnetism, i.e. all spins are canted out of the plane by a small angle. This interaction does not appear in all cuprate antiferromagnets. In $\mathrm{Sr}_{2} \mathrm{CuO}_{2} \mathrm{Cl}_{2}$, for instance, the weak ferromagnetic moment is not registered. In our model we left out this phenomena, since our 
earlier results concerning the temperature law of the magnetization reduction [7] suggested the dominant presence of the gapless excitations leading to the DM interaction gap small enough to be excluded. The limitations imposed to our model by the negligence of this interaction will be discussed later.

\section{Magnetic susceptibility in random-phase approximation}

The GF method used here is based on the temporal Fourier transform of the doubletime temperature spin Green's functions [12], defined as

$$
\langle\langle\hat{A} \mid \hat{B}\rangle\rangle_{\omega}=\frac{1}{2 \pi} \int_{-\infty}^{+\infty} \theta\left(t-t^{\prime}\right)\left\langle\left[\hat{A}(t), \hat{B}\left(t^{\prime}\right)\right]\right\rangle e^{i \omega\left(t-t^{\prime}\right)} d\left(t-t^{\prime}\right) .
$$

The initial point in the calculation will be the equations of motion for the Green's functions $\left\langle\left\langle\hat{S}_{\boldsymbol{n}_{a}}^{+(a)} \mid \hat{B}\right\rangle\right\rangle_{\omega}$ and $\left\langle\left\langle\hat{S}_{\boldsymbol{n}_{b}}^{-(b)} \mid \hat{B}\right\rangle\right\rangle_{\omega}$, obtained by the standard procedure from the Hamiltonian (1), where $\hat{B}$ stands for the arbitrary spin operator component and will be specified later in order to obtain requested Green's functions. The higher-order Green's functions that enter these equations are decoupled using the random-phase approximation [12], which is defined by the relation

$$
\left\langle\left\langle\hat{S}_{\boldsymbol{g}}^{z} \hat{S}_{\boldsymbol{f}}^{+} \mid \hat{B}\right\rangle\right\rangle \stackrel{\boldsymbol{g} \neq \boldsymbol{f}}{\longrightarrow}\left\langle\hat{S}^{z}\right\rangle\left\langle\left\langle\hat{S}_{\boldsymbol{f}}^{+} \mid \hat{B}\right\rangle\right\rangle
$$

This decoupling scheme consists in neglecting the correlations between the longitudinal and transversal spin components at the different lattice sites, whereby the operator $\hat{S}_{\boldsymbol{g}}^{z}$ is replaced by its mean value $\left\langle\hat{S}^{z}\right\rangle$ which is site-independent due to the translational invariance of the lattice. Hence, after performing the spatial Fourier transformation, we obtain the following system of the algebraic equations for the spatio-temporal Fourier transforms of the double-time spin Green's functions (hereafter $\langle\langle\hat{A} \mid \hat{B}\rangle\rangle \equiv\left\langle\langle\hat{A} \mid \hat{B}\rangle_{\boldsymbol{k}_{, \omega}}\right.$ ):

$$
\begin{gathered}
\left(E-\epsilon_{1}\right)\left\langle\left\langle\hat{S}^{+(a)} \mid \hat{B}\right\rangle\right\rangle-\widetilde{J}_{1}(\boldsymbol{k})\left\langle\left\langle\hat{S}^{-(b)} \mid \hat{B}\right\rangle\right\rangle=\frac{i \hbar}{2 \pi}\left\langle\left[\hat{S}^{+(a)}, \hat{B}\right]\right\rangle, \\
\widetilde{J}_{2}(\boldsymbol{k})\left\langle\left\langle\hat{S}^{+(a)} \mid \hat{B}\right\rangle\right\rangle+\left(E+\epsilon_{2}\right)\left\langle\left\langle\hat{S}^{-(b)} \mid \hat{B}\right\rangle\right\rangle=\frac{i \hbar}{2 \pi}\left\langle\left[\hat{S}^{-(b)}, \hat{B}\right]\right\rangle .
\end{gathered}
$$

Here, we use following notation:

$$
\begin{gathered}
\epsilon_{1 / 2}=\left\langle\hat{S}^{z(b / a)}\right\rangle \widetilde{J}+\left\langle\hat{S}^{z(a / b)}\right\rangle \widetilde{J}(\boldsymbol{k}) \pm g \mu_{B} \mathcal{H} \\
\widetilde{J}_{1 / 2}(\boldsymbol{k})=\left\langle\hat{S}^{z(a / b)}\right\rangle \widetilde{J}^{\prime}(\boldsymbol{k})
\end{gathered}
$$

where

$$
\begin{aligned}
\widetilde{J} & =J z\left(g+\lambda_{\perp}^{a b}\right) \\
\widetilde{J}(\boldsymbol{k}) & =J z\left[\lambda_{2}\left(\gamma_{2}(\boldsymbol{k})+\gamma_{3}(\boldsymbol{k})\right)-2 \lambda_{2}+\lambda_{\perp}^{a a}\left(\gamma_{\perp}^{a a}(\boldsymbol{k})-1\right)\right] \\
\widetilde{J}^{\prime}(\boldsymbol{k}) & =J z\left[\gamma_{1}(\boldsymbol{k})+\lambda_{\perp}^{a b} \gamma_{\perp}^{a b}(\boldsymbol{k})\right] .
\end{aligned}
$$

In the relations $(7), z$ is the number of the in-plane nearest neighbors $(z=4)$, parameters $\lambda$ denote the reduced exchange integrals $\left(\lambda_{2}=J_{2} / J, \lambda_{\perp}^{a b / a a}=J_{\perp}^{a b / a a} / J\right)$, while quantities 
$\gamma(\boldsymbol{k})$ present geometric factors defined as:

$$
\begin{gathered}
\gamma_{1}(\boldsymbol{k})=\frac{1}{z} \sum_{\boldsymbol{\delta}_{1}} \mathrm{e}^{i \boldsymbol{\delta}_{1} \cdot \boldsymbol{k}}=\cos \frac{k_{x} a}{2} \cos \frac{k_{y} b}{2}, \quad \gamma_{2}(\boldsymbol{k})=\frac{1}{z} \sum_{\boldsymbol{\delta}_{2}} \mathrm{e}^{i \boldsymbol{\delta}_{2} \cdot \boldsymbol{k}}=\frac{1}{2}\left(\cos k_{x} a+\cos k_{y} b\right) \\
\gamma_{3}(\boldsymbol{k})=\frac{1}{z} \sum_{\boldsymbol{\delta}_{3}} \mathrm{e}^{i \boldsymbol{\delta}_{3} \cdot \boldsymbol{k}}=\cos k_{x} a \cos k_{y} b, \quad \gamma_{\perp}^{a b}(\boldsymbol{k})=\frac{1}{z} \sum_{\boldsymbol{\delta}_{\perp} \mathrm{e}^{i \boldsymbol{\delta}_{\perp}^{a b}} \cdot \boldsymbol{k}}=\cos \frac{k_{x} a}{2} \cos \frac{k_{z} c}{2} \\
\gamma_{\perp}^{a a}(\boldsymbol{k})=\frac{1}{z} \sum_{\boldsymbol{\delta}_{\perp}^{a a}} \mathrm{e}^{i \boldsymbol{\delta}_{\perp}^{a a} \cdot \boldsymbol{k}}=\cos \frac{k_{y} b}{2} \cos \frac{k_{z} c}{2}
\end{gathered}
$$

The spin-wave spectrum is determined by making use of the system (4). We form the homogeneous system of the algebraic linear equations corresponding to (4) (i.e. we take the right hand side terms of both equations to be zero) and determine the spin-wave spectrum from the condition that this new system has nontrivial solutions. After certain algebraic manipulations, we obtain:

$$
E_{1 / 2} \boldsymbol{k}=\frac{1}{2}\left(\sigma_{b}-\sigma_{a}\right)(\widetilde{J}-\widetilde{J}(\boldsymbol{k})) \pm \frac{1}{2} \sqrt{\left(\sigma_{a}+\sigma_{b}\right)^{2}(\widetilde{J}+\widetilde{J}(\boldsymbol{k}))^{2}-4 \sigma_{a} \sigma_{b}\left[\widetilde{J}^{\prime}(\boldsymbol{k})\right]^{2}}+h,
$$

where we introduce the notation $\left\langle\hat{S}^{z(a / b)}\right\rangle=\sigma_{a / b}$ and $g \mu_{B} \mathcal{H}=h$ for brevity. Now we proceed to calculate sublattice magnetizations defined as:

$$
\sigma_{a / b}=\left\langle\hat{S}^{z(a / b)}\right\rangle=\frac{1}{2}-\frac{1}{N} \sum_{\boldsymbol{k}}\left\langle\hat{S}^{-(a / b)} \hat{S}^{+(a / b)}\right\rangle_{\boldsymbol{k}}
$$

In order to obtain the correlation function $\left\langle\hat{S}^{+(b)} \hat{S}^{-(b)}\right\rangle_{\boldsymbol{k}}$, we have to determine the Green's function $\left\langle\left\langle\hat{S}^{-(b)} \mid \hat{S}^{+(b)}\right\rangle\right\rangle$ from the system (4). To that end, we take $\hat{B} \equiv \hat{S}^{+(b)}$, which yields

$$
\left\langle\left\langle\hat{S}^{-(b)} \mid \hat{S}^{+(b)}\right\rangle\right\rangle=\frac{i \hbar}{2 \pi} 2 \sigma_{b} \frac{1}{E_{1 \boldsymbol{k}}-E_{2 \boldsymbol{k}}}\left(\frac{\epsilon_{1}-E_{1 \boldsymbol{k}}}{E-E_{1 \boldsymbol{k}}}-\frac{\epsilon_{1}-E_{2 \boldsymbol{k}}}{E-E_{2 \boldsymbol{k}}}\right),
$$

Therefrom, by making use of the spectral theorem, we determine the correlation function $\left\langle\hat{S}^{+(b)} \hat{S}^{-(b)}\right\rangle_{\boldsymbol{k}}$, which is then inserted into (12) to obtain magnetization of sublattice $b$ in the following self-consistent form:

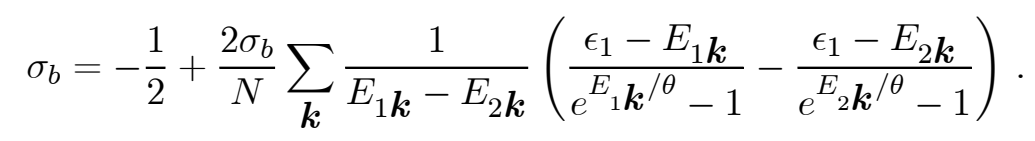

The sublattice $a$ magnetization is determined by the analogous procedure, taking $\hat{B} \equiv$ $\hat{S}^{-(a)}$, which leads to:

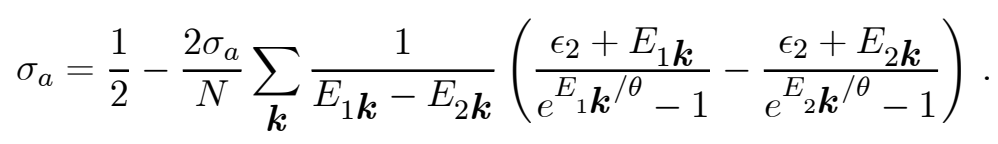

To facilitate the further analysis, it is common to express the sublattice magnetizations in terms of the function $\operatorname{coth} x$, whereafter the expressions (14) and (15) may be written as

$$
\sigma_{b}=-\left[\frac{1}{N} \sum_{\boldsymbol{k}}\left(B \operatorname{coth} \frac{E_{1 \boldsymbol{k}}}{2 \theta}+A \operatorname{coth} \frac{E_{2 \boldsymbol{k}}}{2 \theta}\right)\right]^{-1}
$$


and

$$
\sigma_{a}=\left[\frac{1}{N} \sum_{\boldsymbol{k}}\left(A \operatorname{coth} \frac{E_{1 \boldsymbol{k}}}{2 \theta}+B \operatorname{coth} \frac{E_{2 \boldsymbol{k}}}{2 \theta}\right)\right]^{-1},
$$

where we introduce following notation:

$$
\begin{aligned}
& A=1+\frac{\left(\sigma_{a}+\sigma_{b}\right)(\widetilde{J}+\widetilde{J}(\boldsymbol{k}))}{\sqrt{\left(\sigma_{a}+\sigma_{b}\right)^{2}(\widetilde{J}+\widetilde{J}(\boldsymbol{k}))^{2}-4 \sigma_{a} \sigma_{b}\left[\widetilde{J}^{\prime}(\boldsymbol{k})\right]^{2}}}, \\
& B=1-\frac{\left(\sigma_{a}+\sigma_{b}\right)(\widetilde{J}+\widetilde{J}(\boldsymbol{k}))}{\sqrt{\left(\sigma_{a}+\sigma_{b}\right)^{2}(\widetilde{J}+\widetilde{J}(\boldsymbol{k}))^{2}-4 \sigma_{a} \sigma_{b}\left[\widetilde{J}^{\prime}(\boldsymbol{k})\right]^{2}}}
\end{aligned}
$$

The expressions (16) and (17) present the basis for the calculation of the parallel magnetic susceptibility of the analyzed compound within the framework of the GF method.

\subsection{Magnetic susceptibility in the paramagnetic phase $\left(\theta \geq \theta_{N}\right)$}

The parallel magnetic susceptibility will be calculated according to the procedure proposed for the antiferromagnetic systems in Ref. [13]. Let us first discuss the behavior of that property above the Néel temperature, where the long-range antiferromagnetic order is destroyed due to the thermal fluctuations, i.e. the system passes into its paramagnetic phase. In the presence of the weak external magnetic field $h$, the spins of both sublattices turn in the field direction, leading to

$$
\left|\sigma_{a}\right|=\left|\sigma_{b}\right|=\sigma, \quad\left(\sigma_{b}=-\sigma_{a}\right) .
$$

In that case, expression (18) reduces to $A=B=1$, while the magnon energies (11) read

$$
E_{1 / 2} \boldsymbol{k}=-\sigma\left(\widetilde{J}-\widetilde{J}(\boldsymbol{k}) \mp \widetilde{J}^{\prime}(\boldsymbol{k})\right)+h .
$$

Therefrom the sublattice magnetization becomes:

$$
\sigma=\left[\frac{1}{N} \sum_{\boldsymbol{k}}\left(\operatorname{coth} \frac{-\sigma\left(\widetilde{J}-\widetilde{J}(\boldsymbol{k})-\widetilde{J}^{\prime}(\boldsymbol{k})\right)+h}{2 \theta}+\operatorname{coth} \frac{-\sigma\left(\widetilde{J}-\widetilde{J}(\boldsymbol{k})+\widetilde{J}^{\prime}(\boldsymbol{k})\right)+h}{2 \theta}\right)\right]^{-1}
$$

At high temperatures, we retain only the first term in the power series expansion of the function $\operatorname{coth} x$, yielding:

$$
\sigma \approx\left[\frac{2 \theta}{N} \sum_{\boldsymbol{k}} \frac{2 h-2 \sigma \widetilde{J}+2 \sigma \widetilde{J}(\boldsymbol{k})}{[h-\sigma(\widetilde{J}-\widetilde{J}(\boldsymbol{k}))]^{2}-\sigma^{2}\left[\widetilde{J}^{\prime}(\boldsymbol{k})\right]^{2}}\right]^{-1} .
$$

Substituting $\sigma=\chi_{R P A}(\theta) \cdot h$, previous expression leads to:

$$
\chi_{R P A}(\theta) \approx\left[\frac{4 \theta}{N} \sum_{\boldsymbol{k}} \frac{1-\chi_{R P A}(\theta)(\widetilde{J}-\widetilde{J}(\boldsymbol{k}))}{\left[1-\chi_{R P A}(\theta)(\widetilde{J}-\widetilde{J}(\boldsymbol{k}))\right]^{2}-\chi_{R P A}^{2}(\theta)\left[\widetilde{J}^{\prime}(\boldsymbol{k})\right]^{2}}\right]^{-1}, \theta \geq \theta_{N},
$$


which presents the basis for the further numerical calculations.

\subsection{Magnetic susceptibility in the region of long-range AFM order $\left(\theta \leq \theta_{N}\right)$}

Below the Néel temperature, sublattice magnetizations in the presence of the weak external magnetic field may be presented as

$$
\sigma_{a}=\sigma+\chi_{R P A} \cdot h, \quad \sigma_{b}=\sigma-\chi_{R P A} \cdot h,
$$

where $\sigma$ denotes the magnetization of both sublattices for $h=0$. Thus, the quantities defined by (11) and (18) become

$$
\begin{gathered}
E_{1 / 2} \boldsymbol{k}=E^{\prime}(\boldsymbol{k}) h \pm \frac{1}{2} K(\boldsymbol{k}), \\
A / B=1 \pm \frac{2 \sigma[\widetilde{J}+\widetilde{J}(\boldsymbol{k})]}{K(\boldsymbol{k})},
\end{gathered}
$$

where

$$
E^{\prime}(\boldsymbol{k})=1-\chi_{R P A}[\widetilde{J}-\widetilde{J}(\boldsymbol{k})], \quad K(\boldsymbol{k})=2 \sigma \sqrt{[\widetilde{J}+\widetilde{J}(\boldsymbol{k})]^{2}-\left[\widetilde{J}^{\prime}(\boldsymbol{k})\right]^{2}} .
$$

Further, the term $\operatorname{coth} E_{1 / 2} \boldsymbol{k} / 2 \theta$ appearing in the expressions for the sublattice magnetizations, may be approximated using the identity $\operatorname{coth}(x \pm y)=\frac{1 \pm \operatorname{coth} x \operatorname{coth} y}{\operatorname{coth} x \pm \operatorname{coth} y}$ and retaining only the first term in the power series expansion of $\operatorname{coth} \frac{E^{\prime}(\boldsymbol{k})}{2 \theta} h$, which leads to

$$
\operatorname{coth} \frac{E_{1 / 2(\boldsymbol{k})}}{2 \theta} \approx \pm \operatorname{coth} \frac{K(\boldsymbol{k})}{4 \theta}-h \frac{E^{\prime}(\boldsymbol{k})}{2 \theta} \sinh ^{-2} \frac{K(\boldsymbol{k})}{4 \theta} .
$$

Let us now substitute (16) and (17) together with the notation and approximations introduced in (25)-(28), into the expressions (24). Hence, we obtain

$$
\begin{aligned}
& \sigma+\chi_{R P A} \cdot h=\frac{1}{C\left(1-h \frac{C}{D}\right)} \approx \frac{1}{C}\left(1+h \frac{C}{D}\right), \\
& \sigma-\chi_{R P A} \cdot h=\frac{1}{C\left(1+h \frac{C}{D}\right)} \approx \frac{1}{C}\left(1-h \frac{C}{D}\right),
\end{aligned}
$$

where we use notation

$$
C=\frac{1}{N} \sum_{\boldsymbol{k}} \frac{4 \sigma[\widetilde{J}+\widetilde{J}(\boldsymbol{k})]}{K(\boldsymbol{k})} \operatorname{coth} \frac{K(\boldsymbol{k})}{4 \theta}, \quad D=\frac{1}{N} \sum \boldsymbol{k} \frac{E^{\prime}(\boldsymbol{k})}{\theta} \sinh ^{-2} \frac{K(\boldsymbol{k})}{4 \theta} .
$$

Combining (29) and (30) leads to $\chi_{R P A}=D / C^{2}$ and $\sigma=C^{-1}$. Making use of these two relations leads to:

$$
\chi_{R P A}(\theta)=\frac{\sigma^{2} \frac{1}{N} \sum_{\boldsymbol{k}} \sinh ^{-2} \frac{\sigma \sqrt{[\widetilde{J}+\widetilde{J}(\boldsymbol{k})]^{2}-\left[\widetilde{J^{\prime}}(\boldsymbol{k})\right]^{2}}}{2 \theta}}{\theta+\sigma^{2} \frac{1}{N} \sum_{\boldsymbol{k}}[\widetilde{J}-\widetilde{J}(\boldsymbol{k})] \sinh ^{-2} \frac{\sigma \sqrt{[\widetilde{J}+\widetilde{J}(\boldsymbol{k})]^{2}-\left[\widetilde{J}^{\prime}(\boldsymbol{k})\right]^{2}}}{2 \theta}}, \quad \theta \leq \theta_{N} .
$$


Knowing that $\lim _{\theta \rightarrow \theta_{N}^{-}} \sigma=0$ and retaining only the linear term in the power expansion of $\sinh x$, we obtain:

$$
\chi_{R P A}\left(\theta_{N}\right)=\frac{4 \theta_{N} \frac{1}{N} \sum_{\boldsymbol{k}}\left\{[\widetilde{J}+\widetilde{J}(\boldsymbol{k})]^{2}-\left[\widetilde{J}^{\prime}(\boldsymbol{k})\right]^{2}\right\}^{-1}}{1+4 \theta_{N} \frac{1}{N} \sum_{\boldsymbol{k}}[\widetilde{J}-\widetilde{J}(\boldsymbol{k})]\left\{[\widetilde{J}+\widetilde{J}(\boldsymbol{k})]^{2}-\left[\widetilde{J}^{\prime}(\boldsymbol{k})\right]^{2}\right\}^{-1}},
$$

which presents the parallel magnetic susceptibility at the Néel temperature. The expression for the calculation of the critical temperature within RPA is given in Ref. [5].

\section{Mean-field Theory}

We shall now calculate the parallel magnetic susceptibility in $\mathrm{La}_{2} \mathrm{CuO}_{4}$ in the approximation of the molecular (mean) field, in order to understand the way in which this property is influenced by the short-range spin correlations and quantum spin fluctuations. In the MF approximation magnon energy is wave-vector independent, wherefrom expressions (5) and (11) reduce to

$$
\begin{aligned}
\epsilon_{1 / 2}^{M F A}= & J z\left[\sigma_{b / a}^{M F A}\left(g+\lambda_{\perp}^{a b}\right)+\sigma_{a / b}^{M F A}\left(-2 \lambda_{2}-\lambda_{\perp}^{a a}\right)\right] \pm h, \\
E_{1 / 2}^{M F A} & =\frac{1}{2}\left(\sigma_{b}^{M F A}-\sigma_{a}^{M F A}\right) J z\left[g+\lambda_{\perp}^{a b}+2 \lambda_{2}+\lambda_{\perp}^{a a}\right] \pm \\
& \pm \frac{1}{2}\left(\sigma_{a}^{M F A}+\sigma_{b}^{M F A}\right) J z\left[g+\lambda_{\perp}^{a b}-2 \lambda_{2}-\lambda_{\perp}^{a a}\right]+h .
\end{aligned}
$$

Therefore, expressions (14) and (15) become:

$$
\sigma_{a / b}^{M F A}= \pm \frac{1}{2} \tanh \frac{\sigma_{b / a}^{M F A} J z\left(g+\lambda_{\perp}^{a b}\right) \mp \sigma_{a / b}^{M F A} J z\left(2 \lambda_{2}+\lambda_{\perp}^{a a}\right)+h}{2 \theta} .
$$

In the absence of the external magnetic field sublattice magnetizations do not differ and have value

$$
\sigma^{M F A}=\frac{1}{2} \tanh \frac{\sigma^{M F A} J z\left(g+\lambda_{\perp}^{a b}-2 \lambda_{2}-\lambda_{\perp}^{a a}\right)}{2 \theta} .
$$

Making use of this expression it is simple to calculate $\theta_{N}$ in the MFA, retaining the linear term in the expansion od $\tanh x$ :

$$
\theta_{N}^{M F A}=\frac{1}{4} J z\left(g+\lambda_{\perp}^{a b}-2 \lambda_{2}-\lambda_{\perp}^{a a}\right) .
$$

The analysis of the behavior of magnetic susceptibility in the vicinity of $\theta_{N}$ may be performed by the procedure analogous to that used within RPA. Let us first analyze the paramagnetic phase $\left(\theta \geq \theta_{N}^{M F A}\right)$, where

$$
\sigma_{a}^{M F A}=\sigma^{M F A}, \quad \sigma_{b}^{M F A}=-\sigma^{M F A} .
$$

Substituting (40) and $\sigma^{M F A}=\chi_{M F A}(\theta) \cdot h$ in the expression (37), and using the power series expansion of $\tanh x$, we obtain the following expression for the magnetic susceptibility:

$$
\chi_{M F A}(\theta)=\frac{1}{4}\left[\theta+\frac{1}{4} J z\left(g+\lambda_{\perp}^{a b}+2 \lambda_{2}+\lambda_{\perp}^{a a}\right)\right]^{-1}, \quad \theta \geq \theta_{N}^{M F A} .
$$


(Let us note that expression (23) in the absence of dispersion reduces exactly to (41)).

In the long-range AFM region $\left(\theta \leq \theta_{N}^{M F A}\right)$ in the presence of the weak external field $h$, sublattice magnetizations are, similarly to RPA expressions, given by:

$$
\sigma_{a}^{M F A}=\sigma^{M F A}+\chi_{M F A} \cdot h, \quad \sigma_{b}^{M F A}=\sigma^{M F A}-\chi_{M F A} \cdot h .
$$

Inserting (37) into (42), we obtain:

$$
\begin{gathered}
\sigma^{M F A}+\chi_{M F A} h=\frac{1}{2} \tanh (F+G h), \\
\sigma^{M F A}-\chi_{M F A} h=-\frac{1}{2} \tanh (-F+G h),
\end{gathered}
$$

where $F$ and $G$ are defined as follows:

$$
F=\frac{\sigma^{M F A} J z\left(g+\lambda_{\perp}^{a b}-2 \lambda_{2}-\lambda_{\perp}^{a a}\right)}{2 \theta}, \quad G=\frac{1-\chi_{M F A} J z\left(g+\lambda_{\perp}^{a b}+2 \lambda_{2}+\lambda_{\perp}^{a a}\right)}{2 \theta} .
$$

Combining (43) and (44) and using approximation $\tanh (x+y h) \approx \tanh x+\left(y / \cosh ^{2} x\right) h$, we may easily express the magnetic susceptibility:

$$
\begin{aligned}
& \chi_{M F A}(\theta)=\frac{\left[\cosh \frac{\sigma^{M F A} J z\left(g+\lambda_{\perp}^{a b}-2 \lambda_{2}-\lambda_{\perp}^{a a}\right)}{2 \theta}\right]^{-2}}{4 \theta+J z\left(g+\lambda_{\perp}^{a b}+2 \lambda_{2}+\lambda_{\perp}^{a a}\right)\left[\cosh \frac{\sigma^{M F A} J z\left(g+\lambda_{\perp}^{a b}-2 \lambda_{2}-\lambda_{\perp}^{a a}\right)}{2 \theta}\right]^{-2}}, \\
& \theta \leq \theta_{N}^{M F A} .
\end{aligned}
$$

(Similarly to the comment following (41), we note that expression (32) reduces to (47) within the MFA). Magnetization in the absence of external field $\left(\sigma^{M F A}\right)$ appearing in above relation is given by the self-consistent expression (38), wherefrom it may be determined using the iterative procedure. Finally, the susceptibility at $\theta_{N}$ can be easily calculated from (47), leading to:

$$
\chi_{M F A}\left(\theta_{N}^{M F A}\right)=\frac{1}{4}\left[\theta_{N}^{M F A}+\frac{1}{4} J z\left(g+\lambda_{\perp}^{a b}+2 \lambda_{2}+\lambda_{\perp}^{a a}\right)\right]^{-1} .
$$

Since this value follows also from the expression (41) in the limit $\theta \rightarrow \theta_{N}^{M F A+}$, it is obvious that magnetic susceptibility is continuous function at the Néel temperature.

\section{Spin-wave theory}

Due to the fact that in the theoretical studies $[3,4]$ the magnetic susceptibility analysis is performed also within the framework of LSW theory, we shall do the same in order to compare our results to the ones quoted there. To that end, we shall start from the Hamiltonian (1) and express spin operators as boson ones, using Bloch's approximation. Hence, we obtain the Hamiltonian bilinear in Bose operators which we diagonalize by making use of the well-known "u-v" Bogoliubov transformation. The initial point in calculating parallel magnetic susceptibility will be the expressions for the sublattice magnetizations in 
the presence of the weak external magnetic field acting along $+z$ axis. The magnetization of sublattice $a$ with $N_{a}$ lattice sites, for instance, is determined from the relation

$$
\left\langle\hat{S}_{L S W}^{z(a)}\right\rangle=S-\left\langle\hat{a}_{\boldsymbol{n}}^{\dagger} \hat{a} \boldsymbol{n}\right\rangle=S-\frac{1}{N_{a}} \sum_{\boldsymbol{k}}\left\langle\hat{a}_{\boldsymbol{k}}^{\dagger} \hat{a}_{\boldsymbol{k}}\right\rangle
$$

where the average $\left\langle\hat{a}_{\boldsymbol{k}}^{\dagger} \hat{a}_{\boldsymbol{k}}\right\rangle$ is given by the expression

$$
\left\langle\hat{a}_{\boldsymbol{k}}^{\dagger} \hat{a}_{\boldsymbol{k}}\right\rangle=\frac{u_{\boldsymbol{k}}^{2}}{\exp \left[\beta E_{L S W}^{A}(\boldsymbol{k})\right]-1}+\frac{v_{\boldsymbol{k}}^{2}}{1-\exp \left[-\beta E_{L S W}^{B}(-\boldsymbol{k})\right]} .
$$

Here, magnon energies $E_{L S W}^{A / B}(\boldsymbol{k})$ are defined by

$$
E_{L S W}^{A / B}(\boldsymbol{k})=E_{L S W}(\boldsymbol{k}) \pm h,
$$

where

$$
E_{L S W}(\boldsymbol{k})=\sqrt{\epsilon_{L S W}^{2}(\boldsymbol{k})-I_{L S W}^{2}(\boldsymbol{k})},
$$

while functions $u_{\boldsymbol{k}}$ and $v_{\boldsymbol{k}}$ read

$$
u_{\boldsymbol{k}}=\sqrt{\frac{1}{2}\left[\frac{\epsilon_{L S W}(\boldsymbol{k})}{E_{L S W}(\boldsymbol{k})}+1\right]}, \quad v_{\boldsymbol{k}}=-\sqrt{\frac{1}{2}\left[\frac{\epsilon_{L S W}(\boldsymbol{k})}{E_{L S W}(\boldsymbol{k})}-1\right]} .
$$

In above expressions, we use following notation:

$$
\begin{gathered}
\epsilon_{L S W}(\boldsymbol{k})=J S z\left[(1+\eta)-2 \lambda_{2}+\lambda_{2}\left(\gamma_{2}(\boldsymbol{k})+\gamma_{3}(\boldsymbol{k})\right)+\lambda_{\perp}^{a b}-\lambda_{\perp}^{a a}+\lambda_{\perp}^{a a} \gamma_{\perp}^{a a}(\boldsymbol{k})\right], \\
I_{L S W}(\boldsymbol{k})=J S z\left(\gamma_{1}(\boldsymbol{k})+\lambda_{\perp}^{a b} \gamma_{\perp}^{a b}(\boldsymbol{k})\right) .
\end{gathered}
$$

Using(49), the magnetization of sublattice $a$ becomes:

$$
\begin{aligned}
\left\langle\hat{S}_{L S W}^{z(a)}\right\rangle & =S-\frac{1}{2 N_{a}} \sum_{\boldsymbol{k}}\left[\frac{\epsilon_{L S W}(\boldsymbol{k})}{E_{L S W}(\boldsymbol{k})}\left(\frac{1}{\exp \left(\frac{E_{L S W}^{A}(\boldsymbol{k})}{\theta}\right)-1}+\frac{1}{1-\exp \left(-\frac{E_{L S W}^{B}(\boldsymbol{k})}{\theta}\right)}\right)+\right. \\
& \left.+\left(\frac{1}{\exp \left(\frac{E_{L S W}^{A}(\boldsymbol{k})}{\theta}\right)-1}-\frac{1}{1-\exp \left(-\frac{E_{L S W}^{B}(\boldsymbol{k})}{\theta}\right)}\right)\right]
\end{aligned}
$$

Analogous procedure yields the sublattice $b$ magnetization:

$$
\begin{aligned}
\left\langle\hat{S}_{L S W}^{z(b)}\right\rangle & =S-\frac{1}{2 N_{b}} \sum_{\boldsymbol{k}}\left[\frac{\epsilon_{L S W}(\boldsymbol{k})}{E_{L S W}(\boldsymbol{k})}\left(\frac{1}{\exp \left(\frac{E_{L S W}^{B}(\boldsymbol{k})}{\theta}\right)-1}+\frac{1}{1-\exp \left(-\frac{E_{L S W}^{A}(\boldsymbol{k})}{\theta}\right)}\right)+\right. \\
& \left.+\left(\frac{1}{\exp \left(\frac{E_{L S W}^{B}(\boldsymbol{k})}{\theta}\right)-1}-\frac{1}{1-\exp \left(-\frac{E_{L S W}^{B} A(\boldsymbol{k})}{\theta}\right)}\right)\right]
\end{aligned}
$$

where $N_{a}=N_{b}$. 
In the AFM domain in the presence of external magnetic field it is possible to express the sublattice magnetizations as follows:

$$
\left\langle\hat{S}_{L S W}^{z(a)}\right\rangle=\left\langle\hat{S}_{L S W}^{z}\right\rangle+\chi_{L S W} \cdot h, \quad\left\langle\hat{S}_{L S W}^{z(b)}\right\rangle=\left\langle\hat{S}_{L S W}^{z}\right\rangle-\chi_{L S W} \cdot h,
$$

where $\left\langle\hat{S}_{L S W}^{z}\right\rangle$ presents the magnetization of both sublattices out of magnetic field:

$$
\left\langle\hat{S}_{L S W}^{z(a)}\right\rangle=S-\frac{1}{N_{a}} \sum_{\boldsymbol{k}}\left[\frac{1}{2} \frac{\epsilon(\boldsymbol{k})}{E_{L S W}(\boldsymbol{k})} \operatorname{coth} \frac{E_{L S W}(\boldsymbol{k})}{2 \theta}-\frac{1}{2}\right] .
$$

The parallel magnetic susceptibility may be easily calculated from (57), using relations (55) and (56):

$$
\chi_{L S W} \cdot h=\frac{1}{4 N_{a}} \sum_{\boldsymbol{k}}\left(\operatorname{coth} \frac{E_{L S W}^{B}(\boldsymbol{k})}{2 \theta}-\operatorname{coth} \frac{E_{L S W}^{A}(\boldsymbol{k})}{2 \theta}\right) .
$$

Since we assume that the external field is weak, we may perform the expansion coth $(x+y h)$ $\approx \operatorname{coth} x-\left(y / \sinh ^{2} x\right) h$ in the above expression, leading to the following equation for the magnetic susceptibility:

$$
\chi_{L S W}=\frac{1}{4 \theta} \frac{1}{N_{a}} \sum_{\boldsymbol{k}} \sinh ^{2} \frac{E_{L S W}(\boldsymbol{k})}{2 \theta} .
$$

This expression presents the basis for the numerical analysis performed within the framework of LSW theory.

\section{Analysis of Results}

The numerical analysis of the parallel magnetic susceptibility temperature dependence in $\mathrm{La}_{2} \mathrm{CuO}_{4}$ is first performed within the framework of RPA and MF theory, based upon expressions (23) and (32), and (41) and (47), respectively. Both approaches employ the following set of parameters giving excellent agreement with the experimental value of critical temperature (Ref. [5]), as well as with the magnetization temperature dependence (Refs.[7, 14]): $J=141 \mathrm{meV} ; \lambda_{2}=0.0942 ; g=1+10^{-3} ; \lambda_{\perp}^{a b}=2 \cdot 10^{-4} ; \lambda_{\perp}^{a a}=10^{-4}$. The analysis within the LSW theory, based upon expression (60), is performed for the parameter set chosen to reproduce correctly the experimental magnetization at low temperatures: $J=179 \mathrm{meV} ; \lambda_{2}=0.0615 ; g=1+10^{-3} ; \lambda_{\perp}^{a b}=2 \cdot 10^{-4} ; \lambda_{\perp}^{a a}=10^{-4}$. The results of all three approaches are shown in Fig. 2. In order to facilitate the comparison, the temperature is renormalized to the corresponding critical temperature. Néel temperature in RPA agrees excellently with the experimental value $T_{N}^{\text {exp }} \cong 325 \mathrm{~K}$, while the MF theory predicts much higher value $T_{N}^{M F A} \approx 1330 \mathrm{~K}$ (expression (39)). Within the LSW theory, magnetization is expected to vanish at approximately $700 \mathrm{~K}$. 


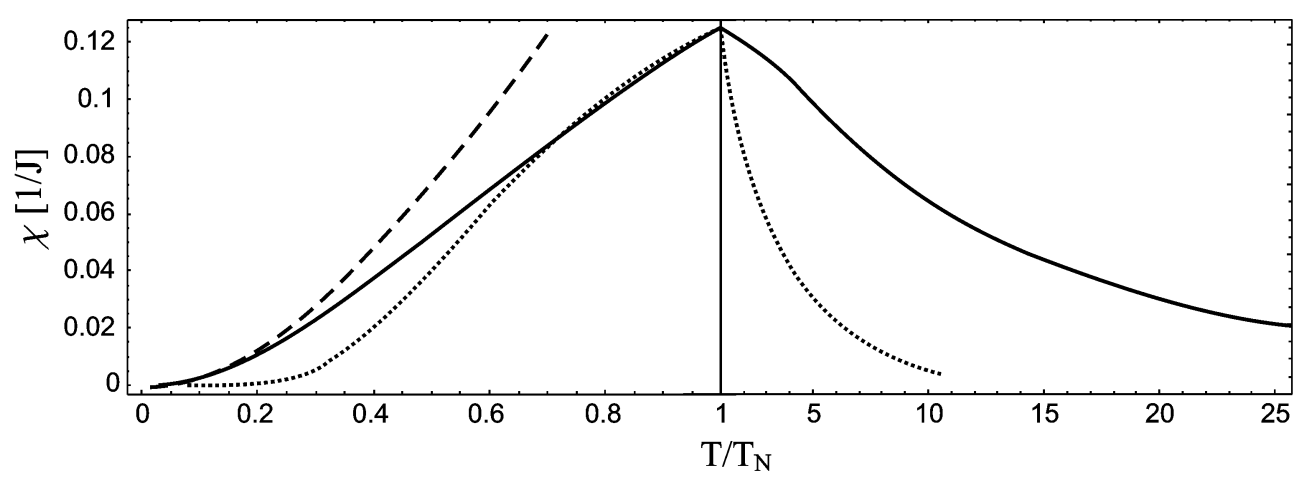

Figure 2. Parallel magnetic susceptibility in $\mathrm{La}_{2} \mathrm{CuO}_{4}$ in RPA (full curve), MFA (dotted curve) and LSW theory (dashed curve).

As can be seen in Fig. 2, in the AFM ordered phase RPA and MFA show close agreement in the vicinity of the Néel temperature $\left(0.7 \leq T / T_{N} \leq 1\right)$. Taking into account that MFA does not include the reduction of the sublattice magnetization caused by the presence of the low-temperature quantum fluctuations, in the low-temperature region RPA and MF results are expected to differ. In that region $\left(T / T_{N} \leq 0.25\right)$ LSW theory shows better agreement with the RPA results. As the temperature grows, the predictions of the LSW theory deviate from RPA results, suggesting the importance of the magnon interactions neglected in the LSW theory, but included in RPA in an approximate way. Above Néel temperature MFA and RPA give essentially different results: in the framework of MFA the susceptibility decreases abruptly, while RPA proposes slow decrease. This is the consequence of the fact that within MFA short-range correlations are neglected.

Since we pretend to determine the domain of applicability and limitations of the model described by Hamiltonian (1), we shall compare our conclusions to those quoted by Tabunshchyk et al. [3, 4]. In Ref. [3], the subject of analysis is one isolated $\mathrm{CuO}_{2}$ plane described by the model which includes the spin-orbit interaction introduced into the effective Hamiltonian by the antisymmetric DM interaction and pseudodipolar symmetric interaction. The presence of these interactions gives rise to long-range order even for the $2 \mathrm{D}$ system (which is, in the case of our model, enabled by the in-plane spin anisotropy). The comparison of our results to those from [3] shows satisfying agreement in the parallel magnetic susceptibility behavior, which exhibits strong dependence on quantum fluctuations and short-range correlations in the wide temperature interval around Néel temperature. The limitation of our model lies in neglecting the weak ferromagnetism of $\mathrm{CuO}_{2}$ planes, precluding us from reproducing the anisotropy of the transversal magnetic susceptibility. Further analysis of our results suggests that the influence of three-dimensionality is expectedly negligible - the calculation performed for the $2 \mathrm{D}$ model gives the results almost indistinguishable from those obtained in three dimensions. That compares favorably to the conclusions from [4] where 3D orthorhombic model is examined, showing that the weak interplanar interactions may be justifiably neglected. In the same Reference, it is quoted that RPA and MFA give the same value for the parallel susceptibility at the Néel temperature, which agrees to the prediction of our model (Fig. 2). Finally, it is interesting to note that in Ref. [4] is also given the study of the approximative 3D tetragonal model, in the framework of which we have as well examined some properties (magnon spectrum, Néel temperature) of the given compound [5]. The conclusion cited in [4] according to 
which orthorhombic $\mathrm{La}_{2} \mathrm{CuO}_{4}$ can be satisfactorily approximated by the tetragonal model agrees to the results of our study.

The comparison to the experimentally determined parallel magnetic susceptibility of the undoped $\mathrm{La}_{2} \mathrm{CuO}_{4}$ is enabled by the results of Lavrov et al. [2]. That paper presents the experimental results for the magnetic susceptibility of the undoped and slightly doped $\mathrm{CuO}_{2}$ planes. Though it had been expected that this property would be completely isotropic in doped regime, the measured anisotropy of magnetic susceptibility suggested that AFM ordered domains survive even in the slightly doped state. For the evaluation of our model it is important to emphasize the qualitative agreement of the results obtained within the framework of RPA to the measured parallel susceptibility of undoped $\mathrm{La}_{2} \mathrm{CuO}_{4}$.

\section{Conclusions}

The aim of our study was the theoretical analysis of the magnetic susceptibility in Q2D cuprate antiferromagnets, especially high- $T_{C}$ superconducting parent compounds, motivated by the permanent interest for that class of compounds, as well as existing theoretical and experimental studies. We performed the thorough analysis of the parallel magnetic susceptibility in undoped $\mathrm{La}_{2} \mathrm{CuO}_{4}$, employing the model with the in-plane spin anisotropy. Three different approaches were implemented: GF method within RPA, LSW theory and MF approximation. The comparison of RPA and MF results have indicated that the quantum spin fluctuations and short range correlations play important role in the behavior of the analyzed property. Results obtained within LSW theory were satisfying in the low-temperature region only, due to the neglecting of the magnon interactions. The comparison to experiment confirmed our conclusion that GF method gives the best description of the parallel susceptibility among the applied approaches.

As far as $\mathrm{YBa}_{2} \mathrm{Cu}_{3} \mathrm{O}_{6}$ is concerned, the complexity of that compound (four-sublattice system) cumbers performing the similarly detailed analysis. Our efforts directed towards obtaining the RPA result met an obstacle already at the point of determination of the magnon energies in the presence of the external magnetic field. The obtained expressions were too complicated to render the further analytical analysis possible. Therefore we performed the MF theory analysis by using the procedure analogous to that invoked for $\mathrm{La}_{2} \mathrm{CuO}_{4}$. The temperature dependence of the parallel magnetic susceptibility resembles the one obtained for $\mathrm{La}_{2} \mathrm{CuO}_{4}$, confirming that $\mathrm{CuO}_{2}$ planes, being the most important common structural feature of the Q2D cuprate antiferromagnets, play dominant role in their magnetic behavior.

Back to $\mathrm{La}_{2} \mathrm{CuO}_{4}$, we may conclude that while our model gives satisfying predictions when it appertains to the parallel magnetic susceptibility, it is not capable of reproducing the transversal one. The limitation of the model lies in neglecting spin-orbit coupling yielding the weak ferromagnetic moment within the $\mathrm{CuO}_{2}$ planes. The analysis based on the effective Hamiltonian with the DM interaction included, in order to obtain the transversal magnetic susceptibility in observed compound, may be the subject of our future studies.

\section{Acknowledgments}

The authors would like to thank Professor Darko Kapor for the critical comments and helpful suggestions. This work was supported by the Serbian Ministry of Education, Science and Technological Development, Project No. OI 171009. 


\section{References}

[1] J. G. Bednorz and K. A. Müller, Z. Physik B 64, 189 (1986)

[2] A. N. Lavrov, Y. Ando, S. Komiya and I. Tsukada, Phys. Rev. Lett. 87, 7007 (2001)

[3] K. V. Tabunshchyk and R. J. Gooding, Phys. Rev. B 71, 214418 (2005)

[4] K. V. Tabunshchyk and R. J. Gooding, J. Phys.: Condens. Matter 17, 6701-6728 (2005)

[5] M. Manojlović, M. Pavkov, M. Škrinjar, M. Pantić, D. Kapor and S. Stojanović, Phys. Rev. B 68, 014435 (2003)

[6] M. Pavkov, M. Škrinjar, D. Kapor, M.Pantić and S.Stojanović, Phys. Rev. B 65, $104512(2002)$

[7] M. S. Rutonjski, S. M. Radošević, M. G. Škrinjar, Pavkov-Hrvojević M. V., D. V. Kapor and M. R. Pantić, Phys. Rev. B 76, 172506 (2007)

[8] M. Manojlović, M. Pavkov, M. Škrinjar, M. Pantić, D. Kapor and S. Stojanović, Phys. Rev. B 71, 132510 (2005)

[9] N. M. Plakida:High-Temperature Cuprate Superconductors, Springer-Verlag, Berlin Heidelberg (2010)

[10] E. Dagotto, Rev. Mod. Phys. 66, 763 (1994)

[11] R. Coldea, S. M. Hayden, G. Aepply, T. G. Perring, C. D. Frost, T. E. Mason, S. W. Cheong and Z. Fisk, Phys. Rev. Lett. 86, 5377 (2001)

[12] S. V. Tyablikov: The Methods in the Quantum Theory of Magnetism, Plenum Press, New York (1967)

[13] L.-P. Lévy: Magnetism and Superconductivity, Springer-Verlag, Berlin Heidelberg (2000)

[14] B. Keimer, A. Aharony, A. Auerbach, R. J. Birgenau, A. Cassanho, Y.Endoh, R. W. Erwin, M. A. Kastner and G. Shirane, Phys. Rev. B 45, 7430 (1992) 